\title{
Prevalence of Soil-Transmitted Helminth and Schistosoma mansoni Infection and Their Associated Factors among Hiruy Abaregawi Primary School Children, Rural Debre Tabor, North West Ethiopia: A Cross-Sectional Study
}

\author{
Lemma Workineh $(\mathbb{D}$, Teklehaimanot Kiros $\mathbb{D}$, Shewaneh Damtie, Tesfaye Andualem $\mathbb{D}$, \\ and Bizualem Dessie
}

Debre Tabor University, College of Medicine and Health Sciences, Department of Medical Laboratory Science, Debre Tabor, Ethiopia

Correspondence should be addressed to Lemma Workineh; lemmiwork@gmail.com

Received 5 August 2019; Revised 6 November 2019; Accepted 26 December 2019; Published 29 January 2020

Academic Editor: Bernard Marchand

Copyright (C) 2020 Lemma Workineh et al. This is an open access article distributed under the Creative Commons Attribution License, which permits unrestricted use, distribution, and reproduction in any medium, provided the original work is properly cited.

\begin{abstract}
Background. In Ethiopia, 25.3 and 12.3 million school-age children are living in soil-transmitted helminth and schistosomiasis endemic areas, respectively. The school children are at risk for both soil-transmitted helminths and Schistosoma mansoni due to juvenile activities like walking barefoot, playing with dirty objects that might be contaminated with feces, and fetching of unclean water for drinking. There are no data that indicate the status of soil-transmitted helminths and Schistosoma mansoni among children at Hiruy Abaregawi primary school. Therefore, the main objective of this study was to determine the prevalence of soil-transmitted helminth and Schistosoma mansoni infection among Hiruy Abaregawi primary school children. Methods. A cross-sectional study was conducted from March to April, 2019, at Hiruy Abaregawi primary school, Rural Debre Tabor, North West Ethiopia. A total of 340 students were included in the study. Informed written consent was obtained from the children's parent. Systematic sampling technique was used to select the children. About 2 grams of stool samples was collected and transported to Debre Tabor University Microbiology and Parasitology Teaching Laboratory to conduct the Kato-Katz technique. Data were analyzed using SPSS version 23. Variables with a $p$ value $<0.05$ were considered statistically significant. Results. The prevalence of soil-transmitted helminths and Schistosoma mansoni was 51/340 (15\%). Among the identified parasites, Ascaris lumbricoides accounts for 28 (8.2\%), hookworm 13 (3.8\%), Trichuris trichiura 4 (1.2\%), and Schistosoma mansoni 6 (1.8\%). In this study, 24 (7\%) of Ascaris lumbricoides-, 11 (3.2\%) of hookworm-, 4 (1.2\%) of Trichuris trichiura-, and 5 (1.5\%) of Schistosoma mansoni-infected children showed light infections and no heavy infection in both soil-transmitted helminths and Schistosoma mansoni was observed. Finger nail trimming status, hand washing before eating, availability of toilet at home, educational level of students, and sex of students were factors associated with soil-transmitted helminth infection. Conclusion and Recommendations. In this study, the low prevalence of soil-transmitted helminths and Schistosoma mansoni was observed. The combination of regular mass deworming program and health information on risk factors should be strengthened for the prevention and control of soil-transmitted helminth infection.
\end{abstract}

\section{Introduction}

Both soil-transmitted helminth (STH) infection and schistosomiasis are considered two of the major public health important neglected tropical diseases (NTDs) and cause chronic malnutrition, stunted growth, cognitive impairment, and anemia $[1,2]$. STH infection is caused by Ascaris lumbri- coides (A. lumbricoides), Trichuris trichiura (T. trichiura), and hookworm whereas schistosomiasis is caused by the medically important Schistosoma species (Schistosoma spp.) such as Schistosoma mansoni (S. mansoni), Schistosoma haematobium (S. haematobium), and Schistosoma japonicum (S. japonicum) [3]. Infection with STHs occurs through accidental ingestion of A. lumbricoides and T. trichiura eggs and 
larval penetration of the skin by hookworm present in contaminated soil while schistosomiasis is transmitted by cercarial skin penetration from fecally contaminated water [4].

STH infection and schistosomiasis infect more than 1.5 billion people and 240 million people, respectively, [5]. Globally, over 267 million preschool-age children and 568 million school-age children live in areas where STHs are intensively transmitted and are in need of treatment and preventive interventions [6]. In sub-Saharan Africa, about 198, 192, 173, and 162 million people are infected with hookworm, Schistosoma spp., A. lumbricoides, and T. trichiura, respectively, $[7,8]$.

In Ethiopia, the number of people living in STH endemic areas is estimated to be 81 million, comprising of 9.1 million preschool-age children, 25.3 million school-age children, and 44.6 million adults whereas about 38.3 million people are living in schistosomiasis endemic areas, comprising of 34.4 million preschool children, 12.3 million school-age children, and 21.6 million adults [9].

In the rural parts of Debre Tabor town, most of the school children are at risk for both STH and S. mansoni infection due to exposure to risky juvenile activities like walking on bare foot, playing with dirty objects that could be contaminated with feces, and drinking of unclean water. There are no data that indicate the prevalence of STH and S. mansoni infection among the school children in the rural parts of Debre Tabor town. So this study will give clue for the stakeholders and policy makers on how to control and prevent STH and S. mansoni infection among the school children in the rural parts of the Debre Tabor town. Therefore, the main objective of this study was to determine the prevalence of STHs and S. mansoni among Hiruy Abaregawi primary school children.

\section{Methods and Materials}

2.1. Study Area and Period. A cross-sectional study was conducted from March to April, 2019, on STH and S. mansoni infections among Hiruy Abaregawi primary school children, rural Debre Tabor, North West Ethiopia. Hiruy Abaregawi primary school is located at the eastern part of rural Debre Tabor town. Debre Tabor is the capital town of the South Gonder zone. It is $102 \mathrm{~km}$ far from Bahir Dar, which is the capital city of Amhara regional sate and $666 \mathrm{~km}$ from Addis Ababa. The total numbers of students learning at the school were 1325. Out of this, 650 and 675 were females and males, respectively.

2.2. Sample Size and Sampling Technique. There are 8 primary schools in Debre Tabor town. Four of them are found in the center of the town and excluded because it is assumed that the prevalence rate decreases due to good sanitation and personal hygiene in the town. Hiruy Abaregawi primary school was randomly selected from the 4 rural Debre Tabor primary schools. Sample size was calculated by using $27.9 \%$ previous prevalence [10]. By using the single proportion formula, $95 \% \mathrm{CI}$, and $5 \%$ margin of error with a $10 \%$ nonresponse rate, 340 students were included in the study. The total number of children from grades 1-8 was 1325 . There were 8 classes and 4 sections in each class in the school. Two sections were selected from each class by using a systematic sampling technique. Then, the number of children sampled from each section was proportionally allocated by dividing the number of students in the selected section to the total number of students in the school, and finally, the proportion was multiplied by the total sample size. Then, after determining the number of students from the selected section, a systematic sampling technique was used to select children.

2.3. Eligibility Criteria. The students who were willing to participate, provided sufficient stool samples, and gave informed assent and consent from their parents were included while the students who had taken helminth chemotherapy before data collection were excluded.

2.4. Parasitological Stool Sample Collection, Processing, and Examination. The school children were given a plastic stool cup and informed to provide about 2 grams of stool sample. Then, the stool samples were preserved with $10 \%$ formalin and packed in a plastic bag to transport to Debre Tabor University Microbiology and Parasitology Teaching Laboratory to conduct the Kato-Katz technique. Two Kato slides per stool sample were prepared using a fixed quantity of sieved $41.7 \mathrm{mg}$ of stool on a punched template [11]. Each stool sample was placed on a newspaper and covered with a nylon screen. The stool was forced through the screen using a plastic spatula, and a portion of the sieved material was filled in the respective template that had been placed on the weighed labeled microscope slides. With a spatula, the surface was leveled and excess stool from the edge of the template hole was carefully removed. Then after, the templates were carefully removed from the slides by lifting it vertically. Finally, the slides were observed within one hour under the microscope at a magnification of $\times 10$ objective.

The total numbers of eggs were expressed as eggs per gram (EPG) of stool. EPG was calculated to classify the infection intensity as light, moderate, and heavy infection [12]. The intensity of STH and S. mansoni infection is defined as light, moderate, and heavy infections, respectively, as follows: A. lumbricoides: 1 EPG to 4999 EPG, 5000 EPG to 49999 EPG, and $\geq 50000$ EPG; T. trichiura: 1 EPG to $999 \mathrm{EPG}$, 1000 EPG to 9999 EPG, and $\geq 10000$ EPG; hookworm: 1 to 1999 EPG, 2000 EPG to 3999 EPG, and $\geq 4000$ EPG; S. mansoni: 1 EPG to $99 \mathrm{EPG}, 100 \mathrm{EPG}$ to $399 \mathrm{EPG}$, and $\geq 400 \mathrm{EPG}$.

Nine laboratory personnel (4 persons for Kato-Katz preparation and 5 persons for microscopic examinations) were involved in the laboratory. Moreover, data on sociodemographic characteristics of the study participants and associated factors of STH and S. mansoni infection were collected by trained health professionals with standardized pretested questionnaires.

2.5. Quality Assurance and Quality Control. In the preanalytical phase, students were oriented on how to collect stool samples; 1 gram of stool was mixed with $3 \mathrm{ml}$ of formalin preservative; sample packaging and transportation, soaking of 
TABLE 1: STH and S. mansoni infections with sociodemographic factors among Hiruy Abaregawi primary school children from March to April, 2019, Debre Tabor Town, North West Ethiopia.

\begin{tabular}{|c|c|c|c|}
\hline \multirow{2}{*}{ Sociodemographic characteristics } & \multicolumn{3}{|c|}{ STH and S. mansoni infection } \\
\hline & Positive & Negative & Total \\
\hline \multicolumn{4}{|l|}{ Sex } \\
\hline Male & $32(16.0 \%)$ & $162(84 \%)$ & $194(57.1 \%)$ \\
\hline Female & $19(13.0 \%)$ & $127(87 \%)$ & $146(42.9 \%)$ \\
\hline \multicolumn{4}{|l|}{ Age } \\
\hline 5-9 & $17(14.5 \%)$ & $100(85.5 \%)$ & $117(34.4 \%)$ \\
\hline $10-14$ & $30(16.0 \%)$ & $159(84.0 \%)$ & $189(55.6 \%)$ \\
\hline $15-18$ & $4(11.7 \%)$ & $30(88.3 \%)$ & $34(10.0 \%)$ \\
\hline \multicolumn{4}{|l|}{ Education level of the students } \\
\hline Grades 1-4 & $32(20.0 \%)$ & $129(80.0 \%)$ & $161(47.4 \%)$ \\
\hline Grades 5-8 & $19(10.6 \%)$ & $160(89.4 \%)$ & $179(52.6 \%)$ \\
\hline \multicolumn{4}{|l|}{ Residence } \\
\hline Rural & $37(15.3 \%)$ & $204(84.7 \%)$ & $241(70.9 \%)$ \\
\hline Urban & $14(14.0 \%)$ & $85(86.0 \%)$ & $99(29.1 \%)$ \\
\hline \multicolumn{4}{|l|}{ Father's educational status } \\
\hline Illiterate & $29(17.0 \%)$ & $140(83.0 \%)$ & $169(49.7 \%)$ \\
\hline $1-8$ & $17(13.0 \%)$ & $115(87.0 \%)$ & $132(38.8 \%)$ \\
\hline High school and above & $5(12.8 \%)$ & $34(87.2 \%)$ & $39(11.5 \%)$ \\
\hline \multicolumn{4}{|l|}{ Mother's educational status } \\
\hline Illiterate & $39(17.6 \%)$ & $182(82.4 \%)$ & $22(65.0 \%)$ \\
\hline $1-8$ & $10(8.8 \%)$ & $103(91.2 \%)$ & $113(33.3 \%)$ \\
\hline High school and above & $1(6.2 \%)$ & $15(93.8 \%)$ & $16(4.7 \%)$ \\
\hline
\end{tabular}

cellophane in glycerol-malachite green solution for 24 hours, and Kato-Katz preparation such as filling stool in the hole of the templates by avoiding overfill and under fill were assured. In the analytical phase, the prepared slides were systematically examined by zigzag form under 10x and 40x microscope objectives. In the postanalytical phase, calculation of egg per gram of stool and documentation were assured.

The performance of Kato-Katz and reagents was checked by positive and negative samples. Finally, ten percent of the examined Kato-Katz slides were randomly selected and reexamined at the end by a qualified laboratory technologist from Amhara Public Health Institute who was blind for the first result. Then, after checking $10 \%$ of the slides, no discordant result was observed.

2.6. Data Management and Analysis. Data were analyzed using SPSS version 23. Descriptive statistics was done to calculate sociodemographic characteristics and the prevalence rate. The associated factors of STH infection were analyzed by binary logistic regression. Firstly, bivariate logistic regression analysis was done, and then to control the possible confounding factors, variables with a $p$ value $<0.2$ were adjusted by multivariate logistic by stepwise variable selection. Finally, variables with a $p$ value $<0.05$ were considered statistically significant.

2.7. Operational Definition. The different prevalence levels are categorized as follows:

Low prevalence: prevalence of less than $50 \%$ for STHs and less than or equal to $10 \%$ for schistosomiasis [1]
Moderate prevalence: prevalence of greater than or equal to $50 \%$ but less than $70 \%$ for STHs and greater than or equal to $10 \%$ but less than $50 \%$ for schistosomiasis

High prevalence: prevalence of greater than or equal to $70 \%$ for STH and greater than or equal to $50 \%$ for schistosomiasis

2.8. Ethical Considerations. Ethical clearance was obtained from Debre Tabor University, College of Medicine and Health Sciences, and permission letter to conduct the study was obtained from the Debre Town education department and Hiruy Abaregawi primary school director office. Additionally, after explaining the importance of the study briefly, an informed written consent was obtained from parents of the school children. Finally, those students who were positive for STHs and S. mansoni were linked to Debre Tabor town health department for treatment by appropriate antihelminthic drugs.

\section{Results}

3.1. Sociodemographic Characteristics of Study Subjects. A total of 340 school children were included in this study. Out of these, 194 (50.6\%) and 146 (49.4\%) were males and females, respectively. The minimum age of the study participants was 5 years, and the maximum age was 18 years with a mean age of 10.6 years. The majority of the study participants, 189 (55.6\%), were in the age group of 10-14 years. The majority of the study subjects comprising of 241 (77\%) were from a rural area (Table 1), and the remaining were from an urban area. 


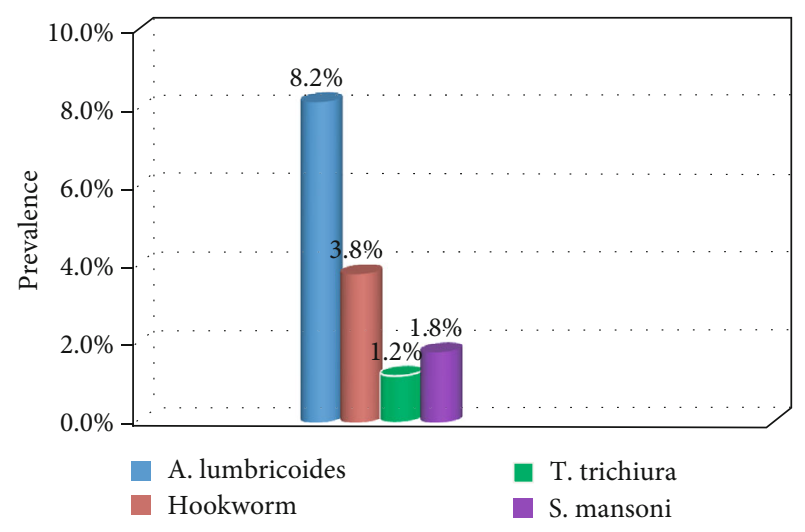

FIgURE 1: Distributions of STH and S. mansoni infections among Hiruy Abaregawi primary school children from March to April, 2019, Debre Tabor town, North West Ethiopia.

3.2. The Prevalence of STH and S. mansoni Infection. Out of 340 school children, 51 students were infected with STHs and S. mansoni with an overall prevalence of $15 \%$ (CI: $11.2 \%-18.8 \%)$. The prevalence of STHs and S. mansoni among males was $16 \%$ while $13 \%$ of females were infected by STHs and S. mansoni. The high prevalence of STHs and S. mansoni was observed in the age group of 10-14 years with the prevalence of $16 \%$, and the least prevalence was observed in the age group of $15-18$ years with $11.7 \%$. About $20 \%$ of students infected by STHs and S. mansoni were from grades 1-4 whereas the least $10.6 \%$ infected children were from grades $5-8$. The students infected by STHs and S. mansoni who were from the rural area were $15.3 \%$ as compared to those who were from the urban area (Table 1).

Among the identified parasites, A. lumbricoides accounted for 28 (8.2\%), hookworm 13 (3.8\%), T. trichiura $4(1.2 \%)$, and S. mansoni 6 (1.8\%) (Figure 1).

3.3. Infection Intensity of STHs and S. mansoni. In this study, $24(7 \%)$ of A. lumbricoides-, 11 (3.2\%) of hookworm-, 4 (1.2\%) of T. trichiura-, and 5 (1.5\%) of S. mansoni-infected children showed light infections (Table 2).

After multiplying the number of eggs per slides by 24 as a factor, the mean intensity of A. lumbricoides, hookworm, $T$. trichiura, and S. mansoni was 1148.6 EPG, 557.5 EPG, 54 EPG, and 68 EPG, respectively.

3.4. Analysis of Factors Associated with STH Infection. All variables were first analyzed by bivariate logistic regression. In bivariate analysis, the age of the respondent, shoewearing habit, hand washing after the toilet, and water source were not associated with STH infection $(p>0.05)$. However, the educational level of the students, residence, trimming of finger nail status, hand washing before eating a meal, and latrine availability were factors associated with STH infection $(p<0.05)$ (Table 3).

All bivariate results that had a $p$ value $<0.2$ were subjected to a multivariate logistic regression model to control possible confounding factors. After adjustment, the shoewearing habit was not significant with STH $(p>0.05)$. Female students were 0.41 less likely to be infected by STHs than male students $(\mathrm{AOR}=0.41, \mathrm{CI}=0.010-0.172)$. The odds of being infected by STHs among students from the rural area were 6.7 as compared to those from the urban areas $(\mathrm{AOR}=6.733, \mathrm{CI}=2.119-21.394)$. The children who did not trim their finger nail were 5 times more likely to be infected by STH than those who did. Those students who did not wash their hands before eating a meal were 5.4 more likely to be infected than who washed their hands before eating a meal always $(\mathrm{AOR}=5.496, \mathrm{CI}=2.012-15.012)$, and the students who washed sometimes were 4.5 more likely to be infected than those who washed always $(\mathrm{AOR}=4.506$, $\mathrm{CI}=1.321-15.370$ ). The odds of being infected by STHs in those children who had no latrine at their home were 3.948 as compared to those who had toilet availability $(\mathrm{AOR}=3.948, \mathrm{CI}=1.510-10.325)$ (Table 4$)$.

\section{Discussions}

The finding of this study showed that the overall prevalence STHs and S. mansoni was 15\% (CI: 11.2\%-18.8\%). The prevalence of STHs was $13.2 \%$ giving $8.2 \%(\mathrm{CI}=5.3 \%-11.2 \%)$ of A. lumbricoides, $3.8 \%$ ( $\mathrm{CI}=2.1 \%-6.2 \%)$ of hookworm, and $1.2 \%$ (CI $=0.3 \%-2.4 \%)$ of $T$. trichiura. The prevalence of $A$. lumbricoides and T. trichiura in this study was similar to the study conducted in Libo kem district [10] with a prevalence of $11 \%$ and $1.6 \%$, respectively.

Moreover, this study was different from other previous studies such as $39.8 \%$ of A. lumbricoides and $6.1 \%$ of $T$. trichiura among primary school children, in North Gonder [13]; $0.2 \%, 14.75 \%$, and $0.2 \%$ of $A$. lumbricoides, hookworm, and T. trichiura, respectively, in Dera district [14]; $12.7 \%$ of A. lumbricoides, $43.4 \%$ of hookworm, and $10 \%$ of T. trichiura, in Zegie peninsula [15]; 31.8\% of hookworm, $29.4 \%$ of A. lumbricoides, and $3.1 \%$ of T. trichiura, in Lake Tana [16]; $37.1 \%$ of hookworm, $16.1 \%$ A. lumbricoides, and $0.8 \%$ of T. trichiura, in Sanja town [17]; 2.6\% of A. lumbricoides, $2.85 \%$ of hookworm, $2.6 \%$ of T. trichiura, in Tach Armachiho district [18]; and 33.3\% of hookworm, $15.5 \%$ of A. lumbricoides, and $1.6 \%$ of T. trichiura, in Motta town [19].

The variations in the prevalence of STHs between the current study and previous study might be due to the difference in sanitation status, temperature difference, and variation in socioeconomic factors. In this study, the lower prevalence in STHs could be due to 258 (75.8\%) of students who had latrine availability at their home, $305(89.7 \%)$ of students who trimmed their finger nail, and $223(65.5 \%)$ of the students who wore shoes always (Table 3 ). The average temperature in Debre Tabor is $14.8^{\circ} \mathrm{C}$ which could make a low prevalence of STH infection in this study. It is well documented that a warmer temperature facilitates infectivity and development of parasite [20].

In this study, the prevalence of $S$. mansoni was $1.8 \%$ $(\mathrm{CI}=0.6 \%-3.2 \%)$. This finding is consistent with $2.4 \%$ in Dera district [14]. However, it is lower than $14.3 \%$ in Lake Tana [16], 89.9\% in Sanja town [17], 56.6\% in Tach Armachiho district [18], and $29.9 \%$ in Zegie peninsula [15]. The difference could be due to low risk factors like absence of a water body for swimming and irrigational activities in the study area. Moreover, the high-altitude nature of Debre 
TABLE 2: Intensity of STH and S. mansoni infections among Hiruy Abaregawi primary school children from March to April, 2019, Debre Tabor town, North West Ethiopia.

\begin{tabular}{|c|c|c|c|c|}
\hline Infection intensity & $\begin{array}{c}\text { A. lumbricoides } \\
\text { No. (\%) }\end{array}$ & $\begin{array}{c}\text { Hookworm } \\
\text { No. }(\%)\end{array}$ & $\begin{array}{l}\text { T. trichiura } \\
\text { No. }(\%)\end{array}$ & $\begin{array}{l}\text { S. mansoni } \\
\text { No. }(\%)\end{array}$ \\
\hline Light infection & $24(7.0)$ & $11(3.2)$ & $4(1.2)$ & $5(1.5)$ \\
\hline Moderate infection & $4(1.2)$ & $2(0.6)$ & $0(0.0)$ & $1(0.3)$ \\
\hline Heavy infection & $0(0.0)$ & $0(0.0)$ & $0(0.0)$ & $0(0)$ \\
\hline Total positive & $28(8.2)$ & $13(3.8)$ & $4(1.2)$ & $6(1.8)$ \\
\hline Negative & $312(91.8)$ & $327(96.2)$ & $336(98.8)$ & $334(98.2)$ \\
\hline Total & $340(100)$ & $340(100)$ & $340(100)$ & $340(100)$ \\
\hline
\end{tabular}

TABLE 3: Bivariate analysis of association factors of STH infection among Hiruy Abaregawi primary school children from March to April, 2019, Debre Tabor town, North West Ethiopia.

\begin{tabular}{|c|c|c|c|c|}
\hline \multirow{2}{*}{ Associate factors of STHs } & \multicolumn{2}{|c|}{ STH infections } & \multirow[t]{2}{*}{ COR $(95 \% \mathrm{CI})$} & \multirow[t]{2}{*}{$p$ value } \\
\hline & Positive & Negative & & \\
\hline \multicolumn{5}{|l|}{ Sex } \\
\hline Male & 28 & 144 & 1 & \\
\hline Female & 17 & 151 & $0.579(0.304-1.103)$ & 0.097 \\
\hline \multicolumn{5}{|l|}{ Age } \\
\hline $5-9$ & 15 & 102 & $1.520(0.413-5.593)$ & 0.529 \\
\hline $10-14$ & 27 & 162 & $1.722(0.492-6.030)$ & 0.395 \\
\hline $15-18$ & 3 & 31 & 1 & \\
\hline \multicolumn{5}{|c|}{ Educational level of the students } \\
\hline Grades 1-4 & 28 & 133 & $2.100(1.003-3.853)$ & 0.034 \\
\hline Grades 5-8 & 17 & 162 & 1 & \\
\hline \multicolumn{5}{|l|}{ Residence } \\
\hline Rural & 35 & 206 & $1.512(0.718-3.187)$ & 0.190 \\
\hline Urban & 10 & 89 & 1 & \\
\hline \multicolumn{5}{|l|}{ Water source } \\
\hline Tap water & 7 & 59 & 1 & \\
\hline Spring & 9 & 80 & $0.948(0.334-2.692)$ & 0.920 \\
\hline Well water & 29 & 156 & $1.567(0.651-3.770)$ & 0.316 \\
\hline \multicolumn{5}{|l|}{ Trimming of finger nail } \\
\hline Yes & 35 & 270 & 1 & \\
\hline No & 10 & 25 & $3.060(1.368-6.961)$ & 0.007 \\
\hline \multicolumn{5}{|c|}{ Hand washing before eating a meal } \\
\hline No & 12 & 36 & $3.239(1.409-7.447)$ & 0.006 \\
\hline Sometimes & 18 & 88 & $2.264(1.088-4.708)$ & 0.029 \\
\hline Always & 15 & 171 & 1 & \\
\hline \multicolumn{5}{|c|}{ Hand washing after using the toilet } \\
\hline No & 16 & 49 & $1.299(0.619-2.716)$ & 0.490 \\
\hline Sometimes & 12 & 95 & $0.719(0.328-1.578)$ & 0.411 \\
\hline Always & 17 & 161 & 1 & \\
\hline \multicolumn{5}{|l|}{ Latrine availability } \\
\hline Yes & 28 & 230 & 1 & \\
\hline No & 17 & 65 & $2.14(1.108-4.167)$ & 0.024 \\
\hline \multicolumn{5}{|l|}{ Shoe-wearing habit } \\
\hline No & 5 & 13 & $2.934(0.970-8.875)$ & 0.057 \\
\hline Sometimes & 13 & 76 & $1.305(0.0640-2.660)$ & 0.464 \\
\hline Always & 17 & 206 & 1 & \\
\hline
\end{tabular}


TABLE 4: Multivariate analysis of association factors of STH infection among Hiruy Abaregawi primary school children from March to April, 2019, Debre Tabor town, North West Ethiopia.

\begin{tabular}{|c|c|c|c|c|}
\hline \multirow{2}{*}{ Associate factors of STHs } & \multicolumn{2}{|c|}{ STH infections } & \multirow{2}{*}{ COR $(95 \% \mathrm{CI})$} & \multirow{2}{*}{$p$ value } \\
\hline & Positive & Negative & & \\
\hline \multicolumn{5}{|l|}{ Sex } \\
\hline Male & 28 & 144 & 1 & \\
\hline Female & 17 & 151 & $0.410(0.010-1.172)$ & 0.000 \\
\hline \multicolumn{5}{|c|}{ Educational level of the students } \\
\hline Grades 1-4 & 28 & 133 & $3.281(1.136-9.479)$ & 0.028 \\
\hline Grades 5-8 & 17 & 162 & 1 & \\
\hline \multicolumn{5}{|l|}{ Residence } \\
\hline Rural & 35 & 206 & $6.733(02.119-9.479)$ & 0.001 \\
\hline Urban & 10 & 89 & 1 & \\
\hline \multicolumn{5}{|l|}{ Trimming of finger nail } \\
\hline Yes & 35 & 270 & 1 & \\
\hline No & 10 & 25 & $5.154(1.300-20.437)$ & 0.020 \\
\hline \multicolumn{5}{|c|}{ Hand washing before eating a meal } \\
\hline No & 12 & 36 & $5.496(2.012-15.012)$ & 0.001 \\
\hline Sometimes & 18 & 88 & $4.506(1.321-15.370)$ & 0.016 \\
\hline Always & 15 & 171 & 1 & \\
\hline \multicolumn{5}{|l|}{ Latrine availability } \\
\hline Yes & 28 & 230 & 1 & \\
\hline No & 17 & 65 & $3.948(1.510-10.325)$ & 0.005 \\
\hline \multicolumn{5}{|l|}{ Shoe-wearing habit } \\
\hline No & 5 & 13 & $3.483(0.836-14.507)$ & 0.086 \\
\hline Sometimes & 13 & 76 & $1.521(0.547-4.230)$ & 0.422 \\
\hline Always & 17 & 206 & 1 & \\
\hline
\end{tabular}

Tabor town could decrease the prevalence of $S$. mansoni in the study area because it is well documented that high altitude decreases the distribution of schistosomiasis [21].

With regard to infection intensity, no heavy infection in both STHs and S. mansoni was observed. About 7\% of $A$. lumbricoides, $3.2 \%$ of hookworm, $1.2 \%$ of $T$. trichiura, and $1.5 \%$ of $S$. mansoni showed light infections whereas $1.2 \%$ of A. lumbricoides, $0.6 \%$ of hookworm, and $0.3 \%$ of S. mansoni showed moderate infections. This finding was different from the study conducted in Tach Armachiho district [18] and in Sanja town [17]. This could be due to the difference in the exposure rate and immunity to reinfection.

In this study, STH infection was associated with finger nail trimming status, hand washing before eating a meal, availability of toilet at home, the educational level of students, and sex of students. These findings were in line with the study done in Tach Armachiho district [18] and Motta town [19].

\section{Conclusion and Recommendations}

In this study, the low prevalence of STHs and S. mansoni was observed. The combination of regular mass deworming program and health information on risk factors should be strengthened for the prevention and control of STH infection.

\section{Data Availability}

The data used to support the findings of this study are included within the article.

\section{Additional Points}

Limitation. We were unable to do an analysis of associated factors with $S$. mansoni due to the low prevalence of $S$. mansoni in the study area.

\section{Conflicts of Interest}

The authors declare that they have no conflicts of interest.

\section{Acknowledgments}

We would like to express great thanks to Hiruy Abaregawi school director and staffs. Our appreciation also goes to all data collectors, and finally, we would like to thank all the school children who participated in this study.

\section{References}

[1] WHO Expert Committee, Prevention and control of schistosomiasis and soil-transmitted helminthiasis, World Health Organization Technical Report Series, 2002. 
[2] S. C. Parija, M. Chidambaram, and J. Mandal, "Epidemiology and clinical features of soil-transmitted helminths," Tropical Parasitology, vol. 7, no. 2, pp. 81-85, 2017.

[3] CDC-ParasitesJuly 2019, http://www.cdc.gov/parasites/.

[4] S. H. Gillespie and R. D. Pearson, Eds., Principles and Practice of Clinical Parasitology, Wiley, Toronto, 2001.

[5] P. J. Hotez, O. A. Asojo, and A. M. Adesina, "Nigeria: "ground zero" for the high prevalence neglected tropical diseases," PLoS Neglected Tropical Diseases, vol. 6, no. 7, 2012.

[6] WHO, Soil-transmitted helminth infectionsJuly 2019, https:// www.who.int/news-room/fact-sheets/detail/soil-transmittedhelminth-infections.

[7] P. J. Hotez and A. Kamath, "Neglected tropical diseases in subSaharan Africa: review of their prevalence, distribution, and disease burden," PLoS Neglected Tropical Diseases, vol. 3, no. 8, p. e412, 2009.

[8] P. Steinmann, J. Keiser, R. Bos, M. Tanner, and J. Utzinger, "Schistosomiasis and water resources development: systematic review, meta- analysis, and estimates of people at risk," The Lancet Infectious Diseases, vol. 6, no. 7, pp. 411-425, 2006.

[9] Federal Democratic Republic of Ethiopia Ministry of Health, Second Edition of Ethiopia National Master Plan for Neglected Tropical Diseases, Ethiopian Federal Ministry of Health, Addis Ababa, Ethiopia, 2016.

[10] T. Addisu and A. Asmamaw, "A survey of soil-transmitted helminths infections and Schistosomiasis mansoni among school children in Libo-Kemkem district, northwest Ethiopia: Cross Sectional study," American Journal of Health Research, vol. 3, no. 2, pp. 57-62, 2015.

[11] N. Katz, A. Chaves, and J. Pellegrino, "A simple device for quantitative stool thick-smear technique in schistosomiasis mansoni," The Revista do Instituto de Medicina Tropical de São Paulo, vol. 14, no. 6, pp. 397-400, 1972.

[12] WHO, Prevention and control of intestinal parasitic infections, WHO Technical Report, Geneva, 1987.

[13] B. Mathewos, A. Alemu, D. Woldeyohannes et al., "Current status of soil transmitted helminths and Schistosoma mansoni infection among children in two primary schools in North Gondar, Northwest Ethiopia: a cross sectional study," BMC Research Notes, vol. 7, no. 1, 2014.

[14] M. B. Shiferaw and A. D. Mengistu, "Helminthiasis: hookworm infection remains a public health problem in Dera district, South Gondar, Ethiopia," PLoS One, vol. 10, no. 12, 2015.

[15] M. Abdi, E. Nibret, and A. Munshea, "Prevalence of intestinal helminthic infections and malnutrition among schoolchildren of the Zegie Peninsula, northwestern Ethiopia," Journal of Infection and Public Health, vol. 10, no. 1, pp. 84-92, 2017.

[16] A. A. Bitew, B. Abera, W. Seyoum et al., "Soil-transmitted helminths and Schistosoma mansoni infections in Ethiopian Orthodox Church students around Lake Tana, northwest Ethiopia," PLoS One, vol. 11, no. 5, 2016.

[17] L. Worku, D. Damte, M. Endris, H. Tesfa, and M. Aemero, "Schistosoma mansoni infection and associated determinant factors among school children in Sanja Town, Northwest Ethiopia," Journal of parasitology research, vol. 2014, Article ID 792536, 7 pages, 2014.

[18] M. Yimer, B. Abera, and W. Mulu, "Soil transmitted helminths and Schistosoma mansoni infections in elementary school children at Tach Armachiho district, North-west Ethiopia," Journal of Applied Sciences Research, vol. 2, no. 2, pp. 43-53, 2014.
[19] Mulusew Andualem Asemahagn, "Parasitic infection and associated factors among the primary school children in Motta town, western Amhara, Ethiopia," American Journal of Public Health Research, vol. 2, no. 6, pp. 248-254, 2014.

[20] A. J. Blum and P. J. Hotez, "Global "worming”: climate change and its projected general impact on human helminth infections," PLOS Neglected Tropical Diseases, vol. 12, no. 7, 2018.

[21] T. A. Ghebreyesus, K. H. Witten, A. Getachew et al., "Schistosome transmission, water-resource development and altitude in northern Ethiopia," Annals of Tropical Medicine and Parasitology, vol. 96, no. 5, pp. 489-495, 2002. 